\title{
Comparison of retrieved noctilucent cloud particle properties from Odin tomography scans and model simulations
}

\author{
Linda Megner $^{1}$, Ole M. Christensen ${ }^{1}$, Bodil Karlsson ${ }^{1}$, Susanne Benze ${ }^{1}$, and Victor I. Fomichev ${ }^{2}$ \\ ${ }^{1}$ Department of Meteorology, Stockholm University, Stockholm, Sweden \\ ${ }^{2}$ CRESS, York University, Toronto, Canada \\ Correspondence to: Linda Megner (linda@misu.su.se)
}

Received: 10 January 2016 - Published in Atmos. Chem. Phys. Discuss.: 11 March 2016

Revised: 16 September 2016 - Accepted: 16 September 2016 - Published: 7 December 2016

\begin{abstract}
Mesospheric ice particles, known as noctilucent clouds or polar mesospheric clouds, have long been observed by rocket instruments, satellites and ground-based remote sensing, while models have been used to simulate ice particle growth and cloud properties. However, the fact that different measurement techniques are sensitive to different parts of the ice particle distribution makes it difficult to compare retrieved parameters such as ice particle radius or ice concentration from different experiments. In this work we investigate the accuracy of satellite retrieval based on scattered light and how this affects derived cloud properties. We apply the retrieval algorithm on spectral signals calculated from modelled cloud distributions and compare the results to the properties of the original distributions. We find that ice mass density is accurately retrieved whereas mean radius is often overestimated and high ice concentrations are generally underestimated. The reason is partly that measurements based on scattered light are insensitive to the smaller particles and partly that the retrieval algorithm assumes a Gaussian size distribution. Once we know the limits of the satellite retrieval we proceed to compare the properties retrieved from the modelled cloud distributions to those observed by the Optical, Spectroscopic, and Infrared Remote Imaging System (OSIRIS) instrument on the Odin satellite. We find that a model with a stationary atmosphere, as given by average atmospheric conditions, does not yield cloud properties that are in agreement with the observations, whereas a model with realistic temperature and vertical wind variations does. This indicates that average atmospheric conditions are insufficient to understand the process of noctilucent cloud growth and that a realistic atmospheric variability is crucial for cloud formation and growth. Further, the agreement between results
\end{abstract}

from the model, when set up with a realistically variable atmosphere, and the observations suggests that our understanding of the growth process itself is reasonable.

\section{Introduction}

At the summer polar mesopause, the coldest region on Earth, the temperature drops low enough so that ice particles can form despite the low water content of a few parts per million. These ice clouds, known as noctilucent clouds (NLCs) or polar mesospheric clouds (PMCs), provide a way to monitor changes in this remote region of the atmosphere, where in situ measurements can only be carried out using rockets. NLCs have been observed by the naked eye since the late 19th century (Leslie, 1885) and since the second half of the 20th century, rocket instruments, satellites, lidars and models have been used to develop our understanding of the clouds (e.g. Witt, 1960; Turco et al., 1982; Barth et al., 1983; Hansen et al., 1989).

The different measurement techniques used in remote sensing and for in situ measurements - and even used in particular types of instruments within these categories - make it difficult to compare retrieved parameters such as ice particle radius or ice concentration from different experiments. For example, many in situ rocket measurements are not sensitive to the size of the particles, as long as they are above a certain aerodynamical threshold that is determined by the shape of the instrument and the speed of the rocket (Hedin et al., 2007). Remote sensing instruments like satellites and lidars, on the other hand, are more sensitive to the particles that more efficiently scatter or absorb light, i.e. the particles 
at the larger end of the size distribution. They, in particular the instruments that observe scattered light, are thus rather insensitive to the smaller end of the size distribution. A direct comparison of, for example, the ice concentrations measured by in situ and remote sensing techniques is, therefore, not straightforward.

Even comparisons between individual satellite observations have proven very difficult (Bailey et al., 2015). These difficulties are also due to the fact that different measurement techniques inevitably favour different parts of the size distribution. For instance, an instrument that measures the absorption of light will be sensitive to the total volume of the ice while an instrument that observes scattered light will be sensitive to different regions of the size distributions depending on what scattering angles it observes. If, as earlier studies have indicated, the size distribution were truly Gaussian with a certain width (see e.g. Rapp and Thomas, 2006), then this problem would be easier to overcome, but as will be shown in this study, our model simulations suggest that this is not generally the case.

The size distribution of ice particles in the cloud layer varies with altitude. Models predict that they range from hundreds or thousands of freshly nucleated small particles per cubic centimetre at the mesopause to 10 or fewer more mature particles per cubic centimetre at approximately 81$83 \mathrm{~km}$ (Megner, 2011). This means that the question of which part of the size distribution an instrument is sensitive to is intricately connected to the altitude region the instrument is sensitive to.

In this paper, we therefore first investigate the accuracy of the Odin satellite's retrieval of properties such as ice mass density $\left(m_{i}\right)$, mean radius and total ice concentration. We do this by applying the retrieval algorithm to spectral signals calculated from modelled cloud distributions (which obviously are fully known) and comparing the retrieved results to the properties of the original distributions. After this we proceed to compare the properties retrieved from the modelled cloud distributions to those observed by satellite. We use satellite observations from the Odin tomography modes (Hultgren et al., 2013) for which the satellite's scanning sequence is specifically designed to provide multiple measurements through the same cloud volume, which enables, via tomography, high-resolution altitude and horizontal observations of the NLCs. We use information from both instruments on board the Odin satellite: the Optical, Spectroscopic, and Infrared Remote Imaging System (OSIRIS) instrument (Llewellyn et al., 2004) gives us high-resolution data of the NLCs and the Sub-Millimeter Radiometer (SMR) instrument (Nordh et al., 2003) provides information of the background temperature and water vapour, which in this experiment are used as inputs to our model.

The specific aims of this study are as follows:

1. Identify what part of the size distribution we capture with an OSIRIS-type measurement and to evaluate to what extent retrieved properties, such as mean radius, $m_{i}$ and ice particle concentration, of the sampled volume represent corresponding actual properties.

2. Investigate if our current knowledge of the microphysics (as represented by the CARMA model) is accurate enough to simulate clouds that match our observations and to pinpoint what model input is crucial for simulating representative clouds.

The paper is structured as follows: in Sect. 2 the Odin tomography scans and the retrieval algorithms of OSIRIS and SMR are described. In Sect. 3 the microphysical model is described. Section 4 gives the results of the comparisons and finally Sect. 5 summarises the conclusions.

\section{Odin tomography scans}

Both OSIRIS and SMR observe the atmosphere in the limb geometry: the co-aligned optical axes of both instruments sweep over a selected altitude range in the forward direction as the entire satellite is nodded up and down. During the stratosphere/mesospheric mode, both instruments scan from 7 to $107 \mathrm{~km}$. However, during the tomography mode, only the NLC region of interest, 78 to $90 \mathrm{~km}$, is scanned. This decreases the horizontal distance between subsequent scans and increases the number of lines of sight through a given atmospheric volume, thus enabling the tomographic retrieval of cloud and background atmosphere properties. During the NH10 and NH11 seasons, a total of 180 orbits were performed using the tomographic mode. The orbits were chosen to provide coincident observations with the Aeronomy of Ice in the Mesosphere (AIM) satellite and cover three 3-day periods during each NLC season (Table 1, Hultgren et al., 2013). A tomographic retrieval algorithm is then used to convert the limb-integrated atmospheric line-of-sight properties into local information about cloud properties or the background atmosphere (Christensen et al., 2015; Hultgren and Gumbel, 2014; Hultgren et al., 2013). Using the tomographic algorithm, these local properties can be retrieved between 78 and $87 \mathrm{~km}$ with a horizontal and vertical resolution of $\sim 330$ and $1 \mathrm{~km}$ respectively. For this analysis we use 4 days of tomographic data (76 scans) between 70 and $77^{\circ} \mathrm{N}$ of July 2010 and 2011, where SMR and OSIRIS data are both available. During these days, clouds and background atmosphere were sampled at solar scattering angles of 70 to $100^{\circ}$.

\subsection{OSIRIS retrieval}

The tomographic algorithm transforms the observed OSIRIS limb radiances into the retrieved volume scatter coefficient, a measure of cloud brightness. In contrast to the input limb radiance, which is dependent on tangent altitude, thus containing signals from the fore- and background, the retrieved volume scatter coefficient is a local signal dependent on the vertical dimension altitude and the horizontal dimension Angle 
Along Orbit (AAO). The algorithm used is the Multiplicative Algebraic Reconstruction Technique (MART) based on maximum probability techniques (Hultgren et al., 2013; Hultgren and Gumbel, 2014).

OSIRIS observes scattered sunlight at wavelengths between 277 and $810 \mathrm{~nm}$ with a spectral resolution of approximately $1 \mathrm{~nm}$. For this study, the volume scatter coefficient at specific wavelengths in the UV range $(277.3,283.5,287.8$, 291.2, 294.4, 300.2 and $304.3 \mathrm{~nm}$; see e.g. Karlsson and Gumbel, 2005, for details) is used to retrieve particle sizes from the OSIRIS radiance measurements by fitting the observed spectral signal to tabulated scattering spectra from numerical T-matrix simulations (Baumgarten et al., 2008; Mishchenko and Travis, 1998). Once a particle mode radius is retrieved, ice concentration and ice mass density can be estimated. In accordance with many other satellite retrieval algorithms (e.g. CIPS, SOFIE, SCHIAMACHY, SBUV), a Gaussian particle size distribution with a width that varies by 0.39 times the retrieved mean radius but stays fixed at $16 \mathrm{~nm}$ for radii larger than $40 \mathrm{~nm}$ (Baumgarten et al., 2010), is assumed. Further, the particles are assumed to be oblate spheroids with an axial ratio of 2 (Eremenko et al., 2005).

The retrieval size for mode radius is constrained to $<100 \mathrm{~nm}$. This is because there is more than one solution when fitting the observed signal to the simulated T-matrix spectra for large particles and scattering angles $>90^{\circ}$ (see von Savigny et al., 2005, Fig. 3 for an equivalent issue). The lack of a unique solution makes it impossible to distinguish between particles $>100$ and smaller particles (around $50 \mathrm{~nm}$ ) in the approach we are using. A consequence of this constraint is that the algorithm will select a small mode radius that fits the signal even in the presence of really large particles. Whether this is an acceptable shortcoming in the retrieval algorithm or not is out of the scope of this study; our conclusions are not affected by this constraint.

The PMC microphysical retrieval and resulting uncertainties in cloud brightness and microphysical products are described in detail by Hultgren et al. (2013) and Hultgren and Gumbel (2014). Based on uncertainty in the input radiances, they estimate a typical statistical error in cloud brightness of $10^{-11} \mathrm{~m}^{-1} \mathrm{str}^{-1}$, which is less than $1 \%$ of the typical NLC peak brightness. Propagating the error of the individual radiances through the tomographic retrieval algorithm, statistical uncertainties in mode radius $(\sim \pm 6 \mathrm{~nm}$ throughout all altitudes), ice concentration (from $\pm 1 \mathrm{~cm}^{-3}$ at $81 \mathrm{~km}$ to $\pm 35 \mathrm{~cm}^{-3}$ at $86 \mathrm{~km}$ ) and ice mass density (negligible at lower PMC altitudes, up to $\pm 5 \mathrm{ng} \mathrm{m}^{-3}$ at $86 \mathrm{~km}$ ) are estimated.

\subsection{SMR retrieval}

SMR measures thermal emission from the $557 \mathrm{GHz}$ water vapour line. From this, the concentration of water vapour and temperature can be retrieved in the aforementioned altitude region. This can be achieved as the line is very strong and be- comes optically thick even in the MLT region. The retrieval is done using the non-linear optimal estimation method with a Levenberg-Marquardt iteration scheme. The resulting precision is $0.2 \mathrm{ppmv}$ for water vapour mixing ratio and $2 \mathrm{~K}$ for temperature with a vertical resolution of $2.5 \mathrm{~km}$ and a horizontal resolution of $200 \mathrm{~km}$. The data used in this study are all collected when SMR was operating in frequency mode 13 , as this mode shows the best agreement with other satellite instruments (within $5 \mathrm{~K}$ for temperature and $20 \%$ for water vapour). For further details see Christensen et al. (2015).

\section{CARMA model}

Community Aerosol and Radiation Model for Atmospheres (CARMA) is a microphysical cloud model that originated from a stratospheric aerosol code (Toon et al., 1979; Turco et al., 1979) and was developed to simulate clouds in a variety of environments ranging from the Earth's atmosphere to other planetary atmospheres. It has been used to simulate NLCs in numerous publications (e.g. Asmus et al., 2015; Kiliani et al., 2015; Chandran et al., 2012; Megner, 2011; Megner et al., 2006; Rapp and Thomas, 2006; Merkel et al., 2009; Stevens, 2005; Vergados and Shepherd, 2009; Lübken et al., 2007). As in the majority of these studies, we use the 1-dimensional set-up of the model to simulate microphysical processes such as ice nucleation and growth, sedimentation and vertical transport. Three interactive constituents are simulated: condensation nuclei $(\mathrm{CN})$, ice particles and water vapour. The $\mathrm{CN}$ are assumed to be meteoric smoke particles with a density of $2 \mathrm{~g} \mathrm{~cm}^{-3}$. The number density and size distribution of the $\mathrm{CN}$ are representative of the middle of the NLC season (10 July) at $68^{\circ} \mathrm{N}$ (see Fig. 1 in Megner et al., 2008a). The nucleation is treated in the framework of droplet theory (Fletcher, 1958) where the probability of nucleation depends on the size of the $\mathrm{CN}$ and the contact angle. The contact angle, also known as the wettability, in turn depends on the surface energies between nucleus, ice and air (Fletcher, 1958; Keesee, 1989; Gumbel and Megner, 2009; Megner and Gumbel, 2009). While this quantity remains uncertain, it has been argued that meteoric smoke acts very efficiently as ice nuclei (Roddy, 1984; Rapp and Thomas, 2006) and the contact angle is, therefore, set to 0.95 in agreement with previous studies (Megner, 2011; Megner et al., 2008a; Rapp and Thomas, 2006). Apart from the details mentioned above, our model set-up is similar to that of Rapp and Thomas (2006): the model domain spans from 72 to $102 \mathrm{~km}$ in altitude with a resolution of $0.25 \mathrm{~km}$. The ice particles are considered spherical and the size distributions are evaluated on radius grids consisting of 40 non-equally spaced size bins between 2 to $900 \mathrm{~nm}$. The piecewise parabolic method algorithm (Colella and Woodward, 1984) is used for both vertical advection and deposition growth (advection in particle radius space) with a time step of $100 \mathrm{~s}$. Following Rapp and Thomas (2006) we further use an eddy diffusion profile adapted from the collec- 
(a)

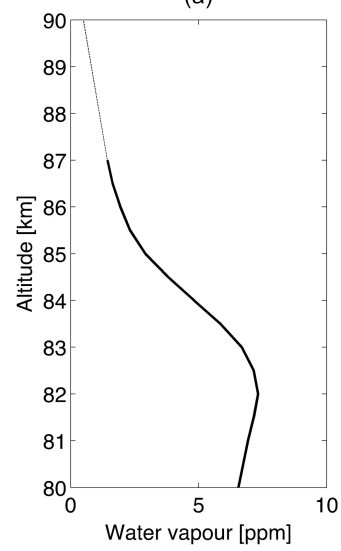

(b)

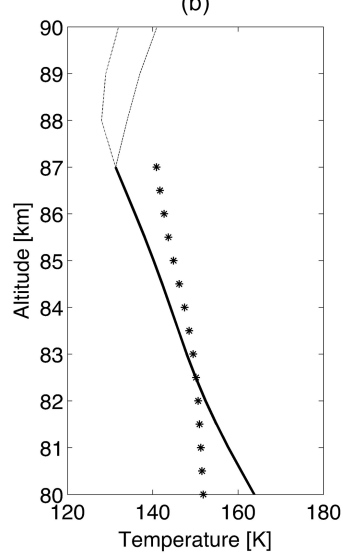

Figure 1. Input data for the No Wave model. (a) Average SMR water vapour (solid line) with a linear extension towards higher altitudes (dashed line). (b) Average SMR temperature (solid line) extended with SABER data (dashed line) and OSIRIS data (dasheddotted line). The stars indicate the average frost point temperature.

tion of turbulence measurements at $69^{\circ} \mathrm{N}$ under polar summer conditions (Lübken, 1997). In all model runs we allow for $5 \mathrm{~h}$ for initialisation after which the next $24 \mathrm{~h}$ are used in the analysis.

\section{Results}

As explained in Sect. 2, the Odin tomography scans give us simultaneous high-resolution observations of ice particles from OSIRIS and water vapour and temperature from SMR. We use these SMR observations as input to the CARMA model and then compare the modelled clouds to those observed by OSIRIS. However, we cannot use the water vapour and temperature profiles from an SMR observation that is made simultaneously to the OSIRIS observation of ice particle properties as the initial state for the model. The reason is that ice growth is not an instantaneous process, i.e. the environment that the clouds grow in is not necessarily the same as the environment they are observed in. For instance the ice growth process itself uses up much of the available water, leading to depletion of water close to the mesopause where the ice grows and enhancement of water where it sublimates. Since we do not have any observations of the history of the atmospheric environment in which the cloud developed we cannot compare a single observed cloud directly to its modelled equivalent. We therefore have to settle for a more statistical approach, by comparing general clouds that are observed by OSIRIS to modelled clouds that have developed in the typical atmospheric environment that SMR observes. In Sects. 4.1 and 4.2 we investigate different ways of creating such a typical environment from the SMR observations and report on the clouds they produce. As presented in the introduction, one main goal of this study is to identify what

part of the size distribution we capture with the OSIRIS-type instrument retrieval and how this is reflected in the retrieved properties such as mean radius, ice mass density and ice concentration. In Sect. 4.3 we investigate this by retrieving sizes from the modelled cloud distributions by applying the same method that we use for the OSIRIS retrievals and comparing the retrieved results to the original distribution. Finally, in Sect. 4.4 we compare the modelled clouds to those observed by OSIRIS.

\subsection{The stationary atmosphere}

In order to generate a typical cloud growth environment from the SMR measurements we select observations that are colocated with the OSIRIS tomography scans where no clouds were present. By selecting only the measurements where no clouds are present we avoid the problem of not accounting for water that is already in the ice phase. We then calculate the average water vapour and temperature profiles and use these fields to drive the model. Since SMR data is only trustworthy up to an altitude of $87 \mathrm{~km}$ we extended the water vapour profile linearly above this altitude, while for the temperature profile we used the SABER profile from Sheese et al. (2011) as shown in Fig. 1. Since SMR does not measure vertical wind we follow Rapp and Thomas (2006) and use a vertical wind profile representative of $69^{\circ} \mathrm{N}$ as given by Berger (2002). The temperature, water vapour and wind profiles in this run are thus stationary. In this model setup, only a very minor $m_{i}$ of maximum $0.03 \mathrm{ng} \mathrm{m}^{-3}$ developed. This is far below the detection threshold of OSIRIS of $5 \mathrm{ng} \mathrm{m}^{-3}$. Hence, if the model is driven by mean atmospheric conditions as measured by the SMR instrument it will not produce visible clouds. The main reason is simply that the small (radii $<\sim 1 \mathrm{~nm}$ ) meteoric smoke particles are not efficient condensation nuclei at a temperature of approximately $131 \mathrm{~K}$ (the mesopause temperature shown in Fig. 1), see Gumbel and Megner (2009). We note that the model set-up used in Rapp and Thomas (2006) does in fact result in observable clouds (and our model reproduces their result given the same input). This is because they use the meteoric smoke distribution of Hunten et al. (1980), which is based on a 1-dimensional model of ablation and recombination of meteoric material and, as such, lacks meridional atmospheric transport. More recently multi-dimensional models have shown that this transport efficiently depletes the summer mesopause of meteoric material resulting in much smaller meteoric smoke particles in this region than what was earlier assumed (Megner et al., 2008b; Bardeen et al., 2008).

The SMR average temperature is declining with altitude up to $87 \mathrm{~km}$, where the measurement quality is diminishing. Thus, there is no information on where exactly the mesopause is. To examine whether a higher (thus colder) mesopause would trigger the model to produce clouds, the temperature profile above the SMR observations was extended to lower temperatures and a higher mesopause using 
the OSIRIS temperatures (Sheese et al., 2011) as shown by the dashed-dotted line in Fig. 1. Although this resulted in a larger $m_{i}$ of maximum $2 \mathrm{ng} \mathrm{m}^{-3}$, it is still below the detection threshold of OSIRIS.

In order to investigate how much colder the atmosphere needs to be for the model to produce clouds, the average temperature profile was reduced in steps of $1 \mathrm{~K}$ and used as input to the model. In order to produce clouds in CARMA of similar $m_{i}$ as the average clouds observed by OSIRIS, the temperature profile had to be reduced by $6 \mathrm{~K}$. However, the particles produced by this model realisation were too large $(150 \mathrm{~nm})$ and their ice concentrations far too small ( $<10$ particles $\mathrm{cm}^{-3}$ throughout the cloud region) compared to the OSIRIS tomography scan observations. Apparently, clouds from this model run were not a realistic representation of the clouds we observe with Odin. We can conclude that a simple shift of the temperature profile towards lower values is not enough to produce realistic NLCs.

Another possibility to facilitate cloud formation is to assume that the CNs are larger or more efficient, so that they can nucleate ice particles at a higher temperature. To test this we first enhanced the contact angle to unity, i.e. perfect wettability (see Sect. 3). This did not have a major effect on the cloud properties and resulted in a maximum ice water density of $0.4 \mathrm{ng} \mathrm{m}^{-3}$, which is still far below the OSIRIS detection limit. However, the $\mathrm{CN}$ distribution is dependent on many uncertain parameters (Megner et al., 2006). For instance, if there is more meteoric influx into the atmosphere, if the CNs are electrically charged (Gumbel and Megner, 2009; Megner and Gumbel, 2009) or if there is more coagulation within the meteor trail than what is generally assumed in models of meteoric coagulation and transport (Megner et al., 2008b; Bardeen et al., 2008), then this could result in a CN distribution that is more efficient for nucleation. Thus we pose the question: what is the number density of efficient CNs required to generate clouds with an $m_{i}$ that agree with the OSIRIS observations? To answer this question we assumed simple mono-sized distributions of particles with radii of $2 \mathrm{~nm}$, i.e. large enough to be efficient $\mathrm{CN}$ at $131 \mathrm{~K}$ (Gumbel and Megner, 2009) but small enough not to rapidly sediment out of the mesopause region. Note, that for simplicity, here we enhance the condensation nuclei efficiency by making the particle larger, but the nucleation efficiency can be enhanced by other means, such as charging of the particles, with equivalent results. By feeding the model mono-sized particle distributions of $10,100,1000$ and 10000 particles $\mathrm{cm}^{-3}$ we determined that approximately 100 efficient $\mathrm{CNs} \mathrm{cm}^{-3}$ was needed to produce an ice mass equivalent to the OSIRIS observations. It should be noted that increasing the number of CNs even more has little effect on the ice mass, as pointed out by Megner (2011); the case with 10000 particles $\mathrm{cm}^{-3}$ gave approximately twice the ice mass compared to the case with 100 particles $\mathrm{cm}^{-3}$. Despite a $\mathrm{CN}$ distribution consisting of 100 particles $\mathrm{cm}^{-3}$ of $2 \mathrm{~nm}$ radii not being considered likely (see Megner, 2011), we nevertheless show the cloud
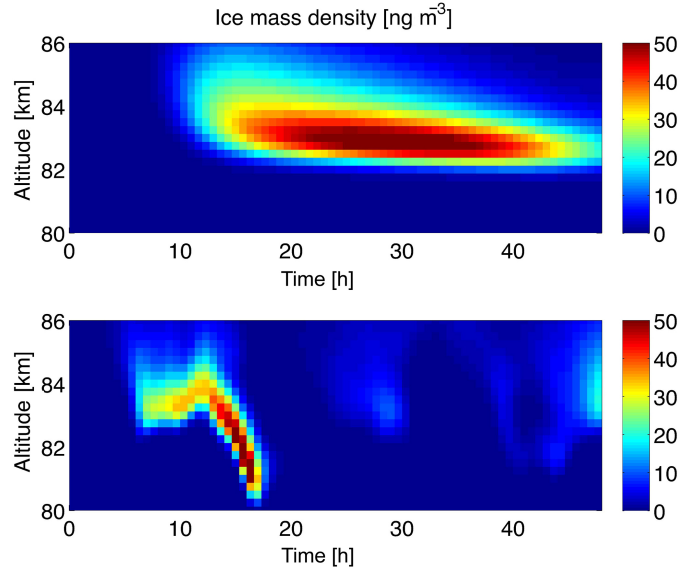

Figure 2. Ice mass density of a cloud generated by the No Wave model set-up (top) and by the Wave set-up (bottom).

generated in this way in Fig. 2 (top panel) as an example of a cloud generated in stationary conditions with a highly efficient $\mathrm{CN}$ distribution. This cloud will be referred to as the "No Wave" cloud.

It is, however, clear that the most straightforward solution to the lack of cloud development in an averaged steady state atmosphere is not that a more efficient size distribution is needed, but simply that the ice particles observed in the real atmosphere are nucleated during the times when the temperature is below average. We will investigate this in the next section.

\subsection{Variable atmosphere}

The mesopause region is characterised by high wave activity (e.g. McLandress et al., 2006). This means that the constant temperature profile achieved by averaging the SMR measurements as described above is not representative. In order to represent the fast temperature variations and vertical winds that give rise to them, we use July temperature and vertical wind fields from July $69^{\circ} \mathrm{N}$ from the extended Canadian Middle Atmosphere Model (CMAM) (Beagley et al., 2010; Fomichev et al., 2002; McLandress et al., 2006) with a high temporal resolution output ( $30 \mathrm{~min})$. In this second set-up of the CARMA model we still use the SMR retrieved mean temperature profile to determine the average conditions, but impose the time resolved CMAM temperature field to represent the temperature variations. In practice this is achieved by adding a temperature shift (constant in time and altitude) to the CMAM data so that the average CMAM temperature profile matches up with the average measured SMR profile. The resulting temperature fields are shown in Fig. 3 and the average temperature profile with the associated temperature variation is shown in Fig. 5a and b. As can be seen, the variations from the CMAM model are fairly similar to those of the SMR data set, especially given that the CMAM variations in- 


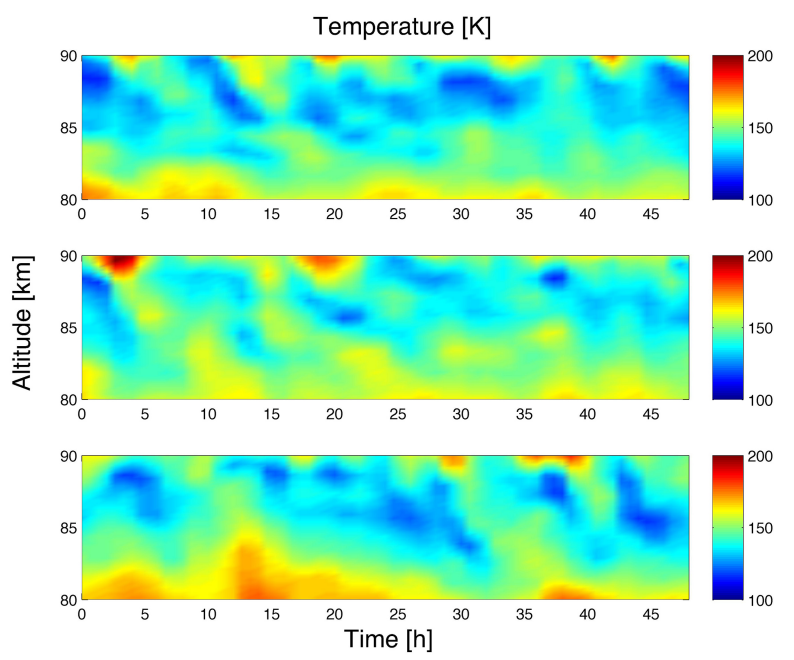

Figure 3. Temperature fields used as input to the Wave model runs.

clude diurnal variations which are not well sampled by SMR since SMR measures predominantly at two local times. The variations of the CMAM model also agree well with observations of daily variations in the summer polar mesopause region (Höffner and Lübken, 2007). Since the vertical wind is intimately connected to the temperature via adiabatic heating/cooling, we use the accompanying CMAM vertical wind field to drive our model simulations (Figs. 4 and 5c, d). The output from the CMAM model was fed into CARMA at time steps of $30 \mathrm{~min}$.

This second model set-up, which includes variations in temperature and winds, resulted in clouds of $m_{i}$ above the OSIRIS detection threshold and, as we shall see, of similar $m_{i}$ as that measured by OSIRIS. An example of a cloud produced in this way can be seen in the lower panel of Fig. 2 . We will refer to these clouds as "Wave" clouds. As the cloud development is somewhat sensitive to the temperature field at the initialisation of the model we perform three simulations: the original, which is initialised at $0 \mathrm{~h}$ (see Figs. 3 and 4), one which is initialised at $10 \mathrm{~h}$ and one that is initialised at $20 \mathrm{~h}$. In all simulations we allow $5 \mathrm{~h}$ for initialisation after which the following $24 \mathrm{~h}$ are included in the analysis.

\subsection{Modelled cloud retrieval}

An important step when comparing the model results to observations is to run the modelled clouds through a similar retrieval process. Since the OSIRIS vertical resolution is broader than that of the model ( $1 \mathrm{~km}$ as opposed to $0.25 \mathrm{~km}$ ), the first step is to linearly average the modelled size distributions over four altitude levels. After that the signal from the modelled size distributions are treated in the same manner as the OSIRIS observations, as described in Sect. 2.1.

In order to investigate how well the retrieval algorithm works, which part of the ice particle size distribution it is sensitive to and how this is reflected in the retrieved prop-
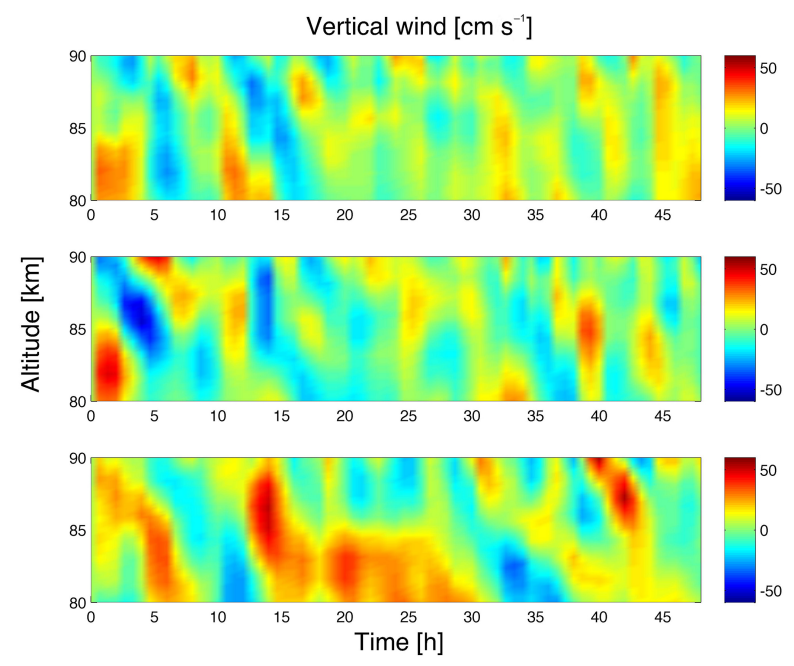

Figure 4. Wind fields used as input to the Wave model runs.

erties, we compare the retrieved modelled clouds to the raw modelled clouds (Fig. 6). As the OSIRIS clouds have been retrieved with an assumption of an axial ratio of 2 , whereas the microphysical treatment of ice particles in the model assumes spheres, i.e an axial ratio of 1 , we show the retrieved properties for both of these assumptions: axial ratio of 2 in black and axial ratio of 1 in grey. For the No Wave clouds (marked with squares) the retrieval is almost entirely independent of axial ratio (indeed the majority of grey squares are hidden by the black squares), whereas the retrieval of the Wave clouds (marked with stars) is somewhat sensitive to the assumption. The reason that the No Wave clouds show almost no sensitivity to the axial ratio may be connected to their size distributions being more well-behaved, as we will discuss later. It is also worth mentioning that the axis ratio not only changes the optical properties of the particles but may also impact their microphysical growth in a way that our CARMA simulation with spherical particles would not capture (Kiliani et al., 2015). Panel a shows that the $m_{i}$ is retrieved rather accurately, for both the No Wave and the Wave clouds, even if the retrieved $m_{i}$ frequently slightly underestimates the volume, especially at higher $m_{i}$. This is encouraging since it indicates that ice mass density is a property we can trust to within approximately $30 \%$.

Figure $6 \mathrm{~b}$ shows that the retrieved mean radius is generally larger than the original mean radius by a factor 2 to 7 for smaller radii. The retrieval of smaller radii $(<20 \mathrm{~nm})$ is worse when an axial ratio of 2 is assumed, which is to be expected given that our model assumes spherical particles, but for larger particles there is no clear difference. Large radii $(\geq 80 \mathrm{~nm})$ are underestimated by the retrieval algorithm. The reason is simply that the retrieval algorithm is constrained to select the smaller radii out of two possible solutions, as described in Sect. 2.1. In practice this prevents the retrieval from retrieving particle sizes above approximately $100 \mathrm{~nm}$. 

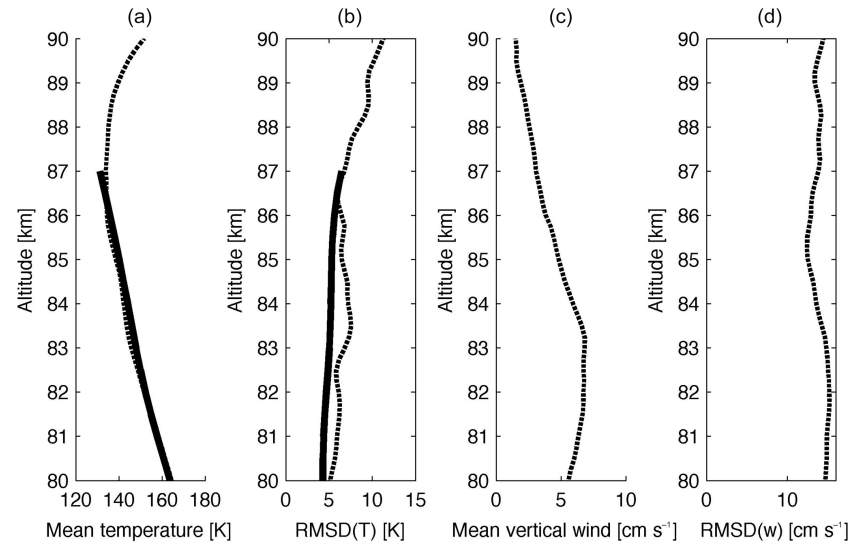

Figure 5. Input to the Wave model set-up (dashed lines). (a) Adjusted average CMAM temperature and (b) temperature variations. The solid lines show the same quantities for the SMR measurements. (c) Average CMAM vertical winds and (d) vertical wind variations.

Figure $6 \mathrm{c}$ shows that high ice concentrations, which generally are associated with small radii at the upper range of the clouds, are greatly underestimated. The underestimation is worse when an incorrect axial ratio (in this case 2) is assumed but can still be as larger than a factor 10 for the retrieval with the correct axis ratio of 1 . For instance, ice concentrations of 1000 particles $\mathrm{cm}^{-3}$ are generally retrieved at approximately 30 particles $\mathrm{cm}^{-3}$ when a ratio of 2 is assumed and as 70 when a ratio of 1 is assumed. It is clear that these large errors in number density arise from the fact that the number density depends on the radius cubed, thus a small error in mean radius will yield a large error in number density.

In order to understand the underestimation of high ice concentrations and the overestimation of small mean radii we study the size distribution. Figure 7 shows a typical example of Wave modelled size distributions at 81 and $84 \mathrm{~km}$ respectively (red line) and the retrieved size distribution using an axial ratio of 2 (black line) and 1 (grey line). Since the retrieval algorithm assumes a Gaussian distribution it obviously cannot retrieve the bimodal distributions that often appear in the model. These multi-peaked distributions arise from the fact that the cold spots produced by atmospheric waves create bursts of newly nucleated particles. These particles then grow and sediment to a region where older and larger cloud particles already exist, resulting in a bimodal size distribution. This effect is more prominent closer to the nucleation region (i.e. the mesopause), thus the size distribution is often less Gaussian at 84 than at $81 \mathrm{~km}$. Due to the nature of light scattering, the retrieval is sensitive mostly to the large end of the particle distribution. This can be seen by the shaded area in Fig. 7, which indicates the particles that contribute the most to the total radiance, thereby being most important for the retrieval (the shaded area contributes with $90 \%$ of the total radiance). It is clear that the radiance is
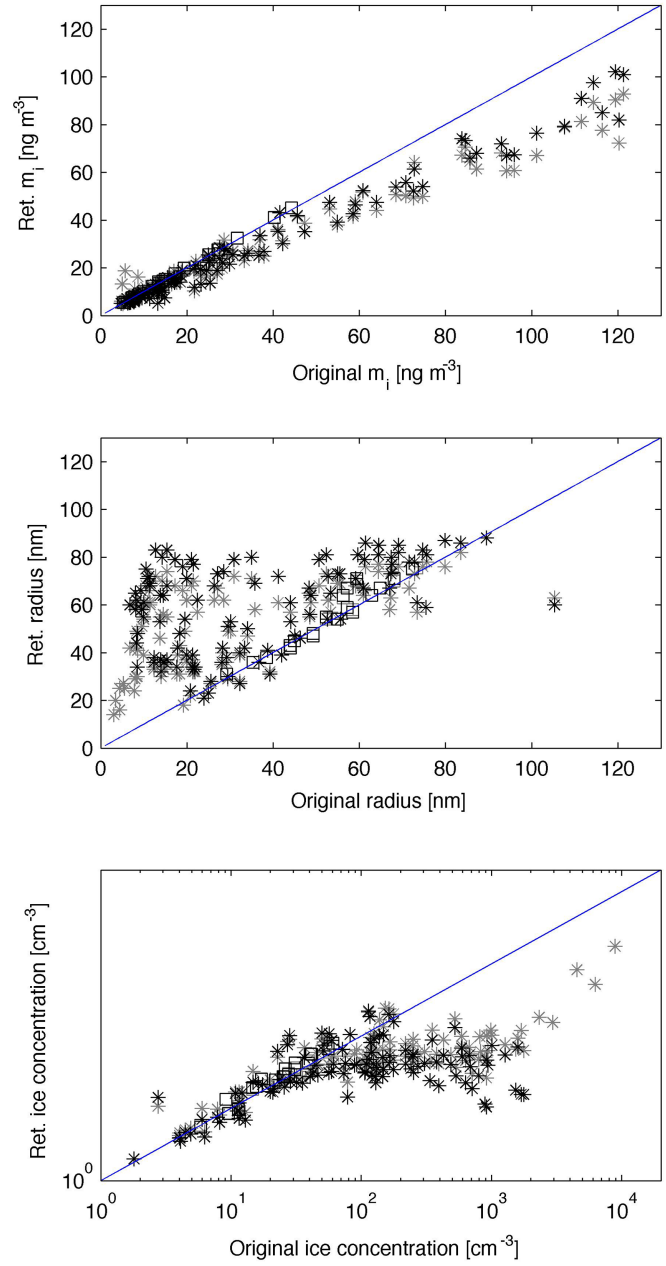

Figure 6. Comparison between properties of the raw modelled clouds and what the OSIRIS retrieval algorithm calculates. Stars indicate Wave clouds and boxes indicate No Wave clouds. Black indicates that oblong particles with an axis ratio of 2 were assumed in the retrieval, and grey indicates that spherical particles were assumed.

dominated by the contribution from the large particles, thus the retrieval will attempt to fit a Gaussian to the larger side of the size distribution. This means that the retrieved mean radius will be larger than the mean radius of the original size distribution, which explains what we saw in the middle and bottom panels of Fig. 6: for smaller radii (generally higher in the cloud) the retrieval often overestimates the mean radius, whereas for larger radii, around 50 to $70 \mathrm{~nm}$, the agreement is better. Furthermore, the total ice concentrations are generally in good agreement when ice concentrations are low (typically lower in the cloud where the size distribution is less bimodal) whereas they are greatly underestimated when ice concentrations are high (typically higher in the cloud, where the particles in the smaller mode are missed by the retrieval).

The No Wave clouds, which are simulated in a stationary environment lacking the cold spots that create the bursts 

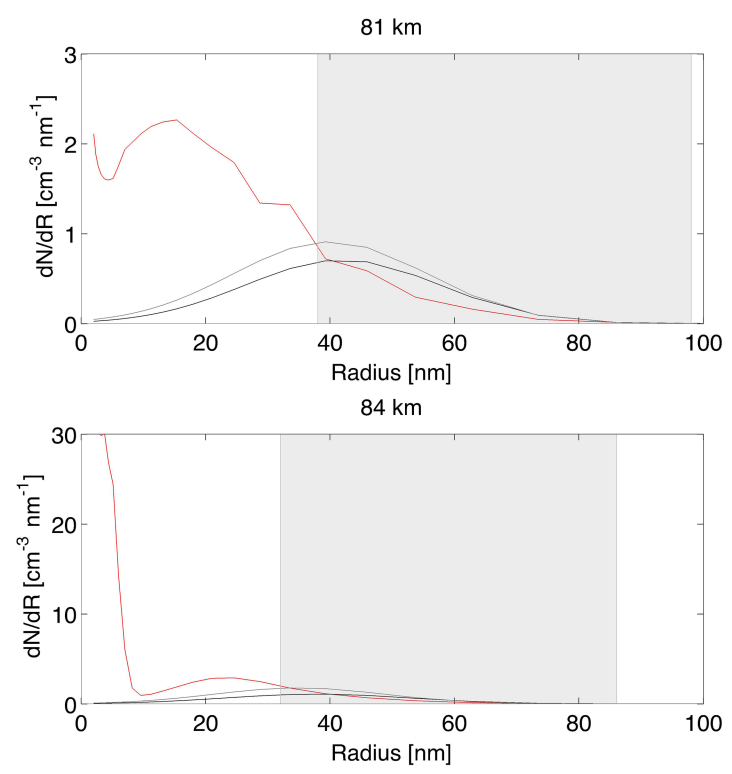

Figure 7. Typical examples of size distributions of the originally modelled clouds (red) and what is retrieved by OSIRIS using an axial ratio of 2 (black) and of 1 (gray) for an altitude of $81 \mathrm{~km}$ (top) and $84 \mathrm{~km}$ (bottom). The grey area indicates the size interval where the top $90 \%$ of the total radiance comes from to give an indication of how the large side of the particle distribution dominates the retrieval.

of fresh ice particles, generally do not show this behaviour, thus their size distributions tend to be more Gaussian (see for instance Rapp and Thomas, 2006). In other words, a stationary atmosphere typically tends to generate Gaussian size distributions whereas temperature variations in the atmosphere generate multi-peaked or less Gaussian-shaped particle size distributions. This is the reason why the properties of the stationary clouds (squares in Fig. 6) in general are better retrieved and their radii/ice concentrations are not overestimated/underestimated in the same way as for clouds generated in a non-stationary atmosphere.

\subsection{Comparison to OSIRIS}

We now move on to comparing the raw and retrieved modelled clouds to the OSIRIS observations. In this section we only show results where an axial ratio of 2 has been assumed in the retrieval, but the figures look similar and the conclusions remain the same if an axis ratio of unity is used.

As mentioned earlier the OSIRIS detection threshold as expressed in $m_{i}$ is approximately $5 \mathrm{ng} \mathrm{m}^{-3}$. In the following we will, therefore, select only the modelled cloud pixels where the retrieved ice mass density is higher than this. However, first we investigate how often this is the case, i.e. the occurrence frequency of clouds above the detection limit. If the model has an accurate description of the atmospheric state then the occurrence frequency in the model should be similar to that of the OSIRIS observations. However, we stress that

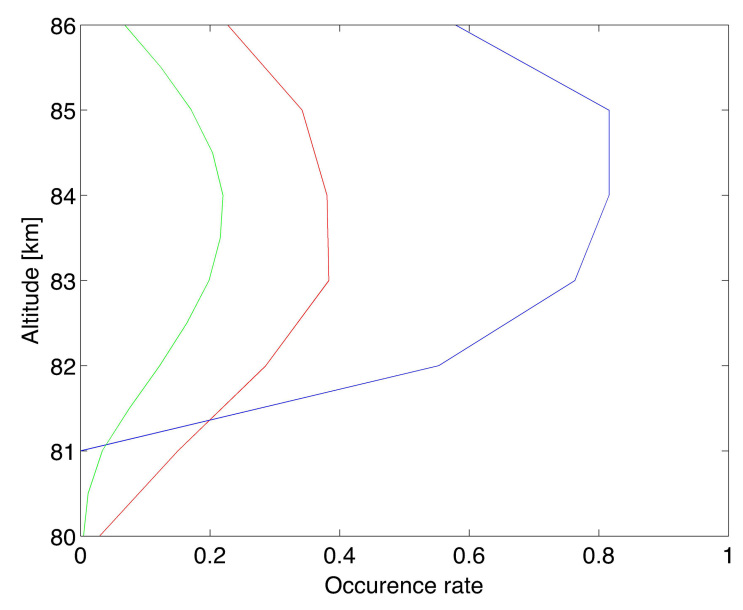

Figure 8. Frequency of occurrence for "Wave" clouds (red) and "No Wave" clouds (blue) and OSIRIS (green).

the occurrence frequency of the model is somewhat dependent on the length of the simulation and the time allowed for initialisation of the model, so that an exact agreement cannot be expected. Figure 8 shows the altitude-dependent occurrence frequency for the OSIRIS observations (in green), the retrieved Wave clouds (in green) and the retrieved No Wave clouds (in blue). While the occurrence frequency of Wave clouds is about twice that of the OSIRIS observations (maximising at 40 and $20 \%$ respectively), the altitudinal distributions of the clouds are similar. The No Wave clouds, on the other hand, show even higher occurrence frequency maximising at $80 \%$ and the altitude extent of the clouds is sharply cut off at $81-82 \mathrm{~km}$.

Figure 9 compares the retrieved properties of the clouds for the Wave clouds (in red), the No Wave clouds (in blue) and the OSIRIS clouds (in green). The thick lines represent the median of all profiles and the shaded fields represent the area between the 10 and 90 percentiles (we choose to plot these instead of the standard deviation since the properties are non-normally distributed). We also plot the median of the raw cloud properties (dashed lines) of the model clouds to show how they differ from the retrieved properties. Panel a shows the retrieved radius, panel $\mathrm{b}$ the ice concentration and panel $\mathrm{c} m_{i}$. When comparing these properties of the clouds, it is important to remember that the No Wave clouds were tuned to produce the correct $m_{i}$ by selecting an appropriate $\mathrm{CN}$ distribution, i.e. the black lines of panel $\mathrm{c}$ have been tuned so that their maximum magnitude corresponds to that of the green lines. One should recall that, without this tuning, the maximum $m_{i}$ that developed was only $0.03 \mathrm{ng} \mathrm{m}^{-3}$, i.e. it would not be visible in the figure. The Wave clouds, on the other hand, have not been tuned to match the OSIRIS results. Despite the lack of tuning, there is a general agreement between the Wave clouds and the OSIRIS observations for all three properties: radius, ice concentration and $m_{i}$, even if the latter is overestimated by the model at lower altitudes. This 

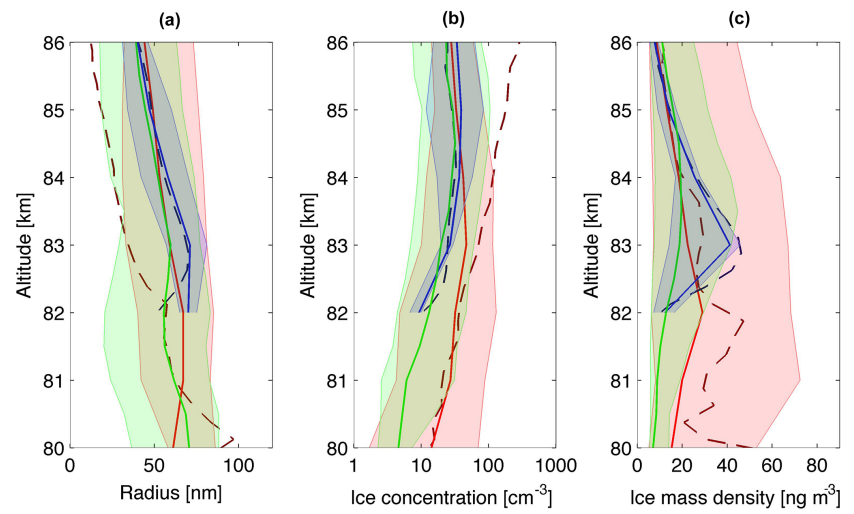

Figure 9. Numeric mean radius, ice concentration and ice mass density for the Wave clouds (in red), the No Wave clouds (in blue) and the OSIRIS clouds (in green). The solid lines in the figure represent the median retrieved properties with shading indicating the interval between the 10 and 90 percentile (percentiles are used instead of standard deviation since the distributions are non-normal). The dashed lines indicate the median of the raw cloud properties (this line is obviously lacking for the OSIRIS clouds).

may be explained by a difference in temperature variability (Fig. 5b), which results in the occurrence of cold temperatures $(<150 \mathrm{~K})$ diminishing faster with altitude for OSIRIS than for CMAM (below $50 \%$ at $83.7 \mathrm{~km}$ for OSIRIS and at $81.8 \mathrm{~km}$ for CMAM).

However, at high altitudes, the raw clouds had much higher ice concentrations and much smaller radii than what was retrieved. For example, at $86 \mathrm{~km}$, the median ice concentration of the raw clouds was 273 particles $\mathrm{cm}^{-3}$ and the median raw radius was $12 \mathrm{~nm}$, but the retrieval shows 28 particles $\mathrm{cm}^{-3}$ of $63 \mathrm{~nm}$ radius. The retrieval becomes marginally better for an assumed axis ratio of $2\left(58\right.$ particles $\mathrm{cm}^{-3}$ of $40 \mathrm{~nm}$ radius, not shown), but the basic problem remains. The reasons for these under-/overestimations were discussed in the previous section. Here we mainly stress that the retrieved Wave clouds may agree with OSIRIS observations, but the measured radius and number densities cloud are far from those of the real clouds.

Clearly the No Wave clouds are restricted to a narrower altitude range than the OSIRIS observations and the Wave clouds (the altitudinal range of the No Wave clouds is insensitive to the choice of $\mathrm{CN}$ distribution and thus it is not affected by the aforementioned tuning). This is easily explained by the static temperature profile, which simply causes conditions that are too warm for clouds to grow below approximately $82.5 \mathrm{~km}$ (see Fig. 1). In the variable atmosphere, on the other hand, the clouds can still exist even when the average temperature (i.e. the temperature used in the static case) is above the frost point, which explains the broader altitudinal extent of the Wave clouds and the OSIRIS observations. Again we see that the retrieval algorithm works

better for the No Wave clouds, which, as earlier explained, is due to them having more Gaussian size distributions.

To summarise, the Wave clouds agree reasonably well with the observations, whereas the clouds from a stationary models are far too weak, and even if they are tuned to the correct $m_{i}$, they appear in a narrower altitude region than the OSIRIS observations show. The differences between the Wave clouds and the observations may be due to the fact that, despite our efforts to create a representative background environment, the corrected CMAM temperature and wind fields are not exact representations of the real background atmosphere in which the clouds have been growing. Unfortunately, due to measurement errors of SMR temperature and water vapour and the lack of vertical wind measurements at the mesopause, the true atmosphere is not exactly known. It may be possible to tune these fields, within the uncertainty of the measurements, to get an even better agreement between the modelled clouds and the observations. However, since these fields are functions of altitude and time (and obviously in the real atmosphere also of horizontal position), there are many free parameters and the limited knowledge of the condensation nuclei adds even more. Therefore, such a tuning would need a significantly larger data set.

\section{Conclusions}

In this paper we have used modelled NLC size distributions to investigate the accuracy of the OSIRIS satellite retrieval algorithm by applying it to our modelled distributions and comparing the retrieved properties to those of the original distributions. We show that ice mass density is well retrieved (within 30\%) whereas mean radius and ice concentrations are much less accurate. The retrieved mean radius is often larger than the actual mean radius, especially for small radii where there can be a difference of up to a factor of 7 . The reason for the inaccuracy is that the retrieval algorithm assumes a Gaussian size distribution and, when faced with the multimodal distributions that often occur in the modelled clouds (thus likely in the real atmosphere), it will attempt to fit a Gaussian to the larger side of the distribution and miss the lower modes, giving an overestimate of the mean radius. Since the size distributions tend to be more multipeaked closer to the nucleation region, this happens more often higher in the cloud where the particles are smaller. At the mesopause we can, therefore, expect large differences in radius and ice concentration between the retrieved and true properties of the clouds. The ice concentration, on the other hand, is retrieved fairly well for small ice concentrations (which generally occur lower in the cloud where the size distributions are more Gaussian), but is typically underestimated by a factor of 10 or more for the high ice concentrations (which generally occur higher in the clouds where the size distributions are more multi-peaked). 
We proceed to compare the retrieved modelled clouds to those of the OSIRIS tomography retrieval runs. The temperature and water vapour fields used to drive the model were inferred from the SMR measurements, which are collocated with the OSIRIS observations of ice particles. We find that driving the model with stationary temperature and wind fields, as given by the average of the SMR measurements, does not yield any observable clouds. In fact, for the model to produce clouds of similar magnitude in ice content to what OSIRIS observes, the average temperature field needs to be reduced by $6 \mathrm{~K}$ and even then the clouds that develop are not representative for the OSIRIS observations in that they consist of very small ice concentrations of too large particles. The reason why no clouds develop in the stationary atmosphere is that the sub-nanometre meteoric smoke particles are too small to be efficient condensation nuclei at the mesopause temperature of $131 \mathrm{~K}$. We show that by increasing the size of the $\mathrm{CN}$, thus making them nucleate more efficiently, it was possible to generate observable clouds. However, in order to generate clouds of ice mass density comparable to the OSIRIS observations, the $\mathrm{CN}$ need to be much larger than what we expect from models of transport and coagulation of meteoric material, or their nucleation properties need to differ significantly from the droplet theory that these models generally assume. Moreover, the altitudinal extent of the clouds produced by the stationary model did not match observations. It is worth pointing out that the stationary model set-up used in Rapp and Thomas (2006) resulted in observable clouds because they used the meteoric smoke distribution of Hunten et al. (1980) which later have been shown to greatly overestimate the number of larger $(>1 \mathrm{~nm}$ radius) meteoric smoke particles at the summer mesopause compared to more advanced models (Megner et al., 2008b; Bardeen et al., 2008). Our stationary model reproduces the results of Rapp and Thomas (2006) if given the same CN distribution as input.

The region of the atmosphere where NLCs develop is far from stationary, as it is heavily influenced by wave activity, which infers large fluctuations in the temperature and wind field, making the actual temperature and winds very different from the average conditions. As a second step we thus imposed more realistic temperature and wind variations on the average SMR fields and used these varying fields as input for the model. Considering the uncertainties of the temperature and wind fields at these altitudes the clouds produced in this way agree reasonably well with OSIRIS observations. Hence, our study suggests that the temperature and wind variations in the summer mesopause region are what drive the formation of the NLC, and that the average fields are not enough to quantitatively describe the process of NLC development. For future model studies, we thus recommend ensuring that not only the averages of the atmospheric fields used to drive the model, but also the variations of these fields are in agreement with observations.
It should be pointed out that there is a clear difference in the size distribution between the clouds modelled using stationary atmospheric conditions and the more realistic clouds where varying temperature and wind field have been used. The former often have more Gaussian size distributions whereas the latter have multimodal size distributions. Since the atmosphere is non-static, the assumption of a Gaussian (or any single mode) distribution should be treated with care. While it may still be justified to use a single mode distribution, simply from the fact that there is a limited number of free parameters one can retrieve using remote sensing techniques, the user of the data should be aware that the ice concentrations and mean radii retrieved in this way are likely not in agreement with what an in situ particle counter would detect.

Finally, we point out that while this study has concentrated on the OSIRIS satellite retrieval algorithm, the main conclusions should be similar for other satellite retrievals that are based on scattering techniques and use the same assumptions for retrieving microphysical parameters.

\section{Data availability}

The CARMA model is widely used and continuously developed by the research community. The version used in this study is (apart from the changes described in the paper) the same as in Rapp et al. (2006). This, and the CMAM model temperature and wind data, can be obtained by request to Linda Megner (linda.megner@misu.su.se).

Acknowledgements. The authors would like to thank their colleagues, in particular the people in the particle size working group, for helpful discussions. Linda Megner was supported by the Swedish Research Council under contract 621-2012-1648, project 1504401. Victor I. Fomichev was supported by the Canadian Space Agency.

Edited by: F.-J. Lübken

Reviewed by: two anonymous referees

\section{References}

Asmus, H., Robertson, S., Dickson, S., Friedrich, M., and Megner, L.: Charge balance for the mesosphere with meteoric dust particles, J. Atmos. Sol.-Terr. Phy., 127, 137-149, doi:10.1016/j.jastp.2014.07.010, 2015.

Bailey, S. M., Thomas, G. E., Hervig, M. E., and Lumpe, J. D.: Comparing nadir and limb observations of polar mesospheric clouds: The effect of the assumed particle size distribution, J. Atmos. Sol.-Terr. Phy., 127, 51-65, doi:10.1016/j.jastp.2015.02.007, 2015.

Bardeen, C. G., Toon, O. B., Jensen, E. J., Marsh, D. R., and Harvey, V. L.: Numerical simulations of the three-dimensional distribution of meteoric dust in the mesosphere and upper stratosphere, J. Geophys. Res., 113, D17202, doi:10.1029/2007JD009515, 2008. 
Barth, C. A., Rusch, D. W., Thomas, R. J., Mount, G. H., Rottman, G. J., Thomas, G. E., Sanders, R. W., and Lawrence, G. M.: Solar Mesosphere Explorer: Scientific objectives and results, Geophys. Res. Lett., 10, 237-240, doi:10.1029/GL010i004p00237, 1983.

Baumgarten, G., Fiedler, J., Lübken, F.-J., and von Cossart, G.: Particle properties and water content of noctilucent clouds and their interannual variation, J. Geophys. Res.-Atmos., 113, D06203, doi:10.1029/2007JD008884, 2008.

Baumgarten, G., Fiedler, J., and Rapp, M.: On microphysical processes of noctilucent clouds (NLC): observations and modeling of mean and width of the particle size-distribution, Atmos. Chem. Phys., 10, 6661-6668, doi:10.5194/acp-10-6661-2010, 2010.

Beagley, S. R., Boone, C. D., Fomichev, V. I., Jin, J. J., Semeniuk, K., McConnell, J. C., and Bernath, P. F.: First multi-year occultation observations of $\mathrm{CO}_{2}$ in the MLT by ACE satellite: observations and analysis using the extended CMAM, Atmos. Chem. Phys., 10, 1133-1153, doi:10.5194/acp-10-1133-2010, 2010.

Berger, U.: Icy particles in the summer mesopause region: Threedimensional modeling of their environment and two-dimensional modeling of their transport, J. Geophys. Res., 107, 1366, doi:10.1029/2001JA000316, 2002.

Chandran, A., Rusch, D. W., Thomas, G. E., Palo, S. E., Baumgarten, G., Jensen, E. J., and Merkel, A. W.: Atmospheric gravity wave effects on polar mesospheric clouds: A comparison of numerical simulations from CARMA 2D with AIM observations, J. Geophys. Res.-Atmos., 117, D20104, doi:10.1029/2012JD017794, 2012.

Christensen, O. M., Eriksson, P., Urban, J., Murtagh, D., Hultgren, K., and Gumbel, J.: Tomographic retrieval of water vapour and temperature around polar mesospheric clouds using OdinSMR, Atmos. Meas. Tech., 8, 1981-1999, doi:10.5194/amt-81981-2015, 2015.

Colella, P. and Woodward, P. R.: The Piecewise Parabolic Method (PPM) for gas-dynamical simulations, J. Comput. Phys., 54, 174-201, doi:10.1016/0021-9991(84)90143-8, 1984.

Eremenko, M. N., Petelina, S. V., Zasetsky, A. Y., Karlsson, B., Rinsland, C. P., Llewellyn, E. J., and Sloan, J. J.: Shape and composition of PMC particles derived from satellite remote sensing measurements, Geophys. Res. Lett., 32, L16S06, doi:10.1029/2005GL023013, 2005.

Fletcher, N. H.: Size Effect in Heterogeneous Nucleation, J. Chem. Phys., 29, 572-576, doi:10.1063/1.1744540, 1958.

Fomichev, V. I., Ward, W. E., Beagley, S. R., McLandress, C., McConnell, J. C., McFarlane, N. A., and Shepherd, T. G.: Extended Canadian Middle Atmosphere Model: Zonal-mean climatology and physical parameterizations, J. Geophys. Res., 107, 4087, doi:10.1029/2001JD000479, 2002.

Gumbel, J. and Megner, L.: Charged meteoric smoke as ice nuclei in the mesosphere: Part 1 - A review of basic concepts, J. Atmos. Sol.-Terr. Phy., 71, 1225-1235, doi:10.1016/j.jastp.2009.04.012, 2009.

Hansen, G., Serwazi, M., and von Zahn, U.: First detection of a noctilucent cloud by lidar, Geophys. Res. Lett., 16, 1445-1448, doi:10.1029/GL016i012p01445, 1989.

Hedin, J., Gumbel, J., and Rapp, M.: On the efficiency of rocketborne particle detection in the mesosphere, Atmos. Chem. Phys., 7, 3701-3711, 10.5194/acp-7-3701-2007, 2007.
Höffner, J. and Lübken, F. J.: Potassium lidar temperatures and densities in the mesopause region at Spitsbergen $\left(78^{\circ} \mathrm{N}\right)$, J. Geophys. Res.-Atmos., 112, D20114, doi:10.1029/2007jd008612, 2007.

Hultgren, K. and Gumbel, J.: Tomographic and spectral views on the lifecycle of polar mesospheric clouds from Odin/OSIRIS, J. Geophys. Res.-Atmos., 119, 14129-14143, doi:10.1002/2014jd022435, 2014.

Hultgren, K., Gumbel, J., Degenstein, D., Bourassa, A., Lloyd, N., and Stegman, J.: First simultaneous retrievals of horizontal and vertical structures of polar mesospheric clouds from Odin/OSIRIS tomography, J. Atmos. Sol.-Terr. Phy., 104, 213 223, 2013.

Hunten, D. M., Turco, R. P., and Toon, O. B.: Smoke and dust particles of meteoric origin in the Mesosphere and Stratosphere, J. Atmos. Sci., 37, 1342-1357, 1980.

Karlsson, B. and Gumbel, J.: Challenges in the limb retrieval of noctilucent cloud properties from Odin/OSIRIS, Adv. Space Res., 36, 935-942, doi:10.1016/j.asr.2005.04.074, 2005.

Keesee, R. G.: Nucleation and particle formation in the upper atmosphere, J. Geophys. Res.-Atmos., 94, 14683-14692, doi:10.1029/JD094iD12p14683, 1989.

Kiliani, J., Baumgarten, G., Lübken, F.-J., and Berger, U.: Impact of particle shape on the morphology of noctilucent clouds, Atmos. Chem. Phys., 15, 12897-12907, doi:10.5194/acp-1512897-2015, 2015.

Leslie, R.: Sky glows, Nature, 32, p. 245, 1885.

Llewellyn, E. J., Lloyd, N. D., Degenstein, D. A., Gattinger, R. L., Petelina, S., and Bourassa, A. E.: The OSIRIS instrument on the Odin spacecraft, Can. J. Phys., 82, 411-422, doi:10.1139/p04005, 2004.

Lübken, F. J.: Seasonal variation of turbulent energy dissipation rates at high latitudes as determined by in situ measurements of neutral density fluctuations, J. Geophys. Res.-Atmos., 102, 13441-13456, doi:10.1029/97JD00853, 1997.

Lübken, F.-J., Rapp, M., and Strelnikova, I.: The sensitivity of mesospheric ice layers to atmospheric background temperatures and water vapor, Adv. Space Res., 40, 794-801, doi:10.1016/j.asr.2007.01.014, 2007.

McLandress, C., Ward, W. E., Fomichev, V. I., Semeniuk, K., Beagley, S. R., McFarlane, N. A., and Shepherd, T. G.: Large-scale dynamics of the mesosphere and lower thermosphere: An analysis using the extended Canadian Middle Atmosphere Model, J. Geophys. Res., 111, D17111, doi:10.1029/2005JD006776, 2006.

Megner, L.: Minimal impact of condensation nuclei characteristics on observable Mesospheric ice properties, J. Atmos. Sol.-Terr. Phy., 73, 2184-2191, doi:10.1016/j.jastp.2010.08.006, 2011.

Megner, L. and Gumbel, J.: Charged meteoric particles as ice nuclei in the mesosphere: Part 2, J. Atmos. Sol.-Terr. Phy., 71, 12361244, doi:10.1016/j.jastp.2009.05.002, 2009.

Megner, L., Rapp, M., and Gumbel, J.: Distribution of meteoric smoke - sensitivity to microphysical properties and atmospheric conditions, Atmos. Chem. Phys., 6, 4415-4426, doi:10.5194/acp-6-4415-2006, 2006.

Megner, L., Gumbel, J., Rapp, M., and Siskind, D. E.: Reduced meteoric smoke particle density at the summer pole - Implications for mesospheric ice particle nucleation, Adv. Space Res., 41, 4149, doi:10.1016/j.asr.2007.09.006, 2008a. 
Megner, L., Siskind, D. E., Rapp, M., and Gumbel, J.: Global and temporal distribution of meteoric smoke: A two-dimensional simulation study, J. Geophys. Res.-Atmos., 113, D03202, doi:10.1029/2007JD009054, 2008b.

Merkel, A. W., Marsh, D. R., Gettelman, A., and Jensen, E. J.: On the relationship of polar mesospheric cloud ice water content, particle radius and mesospheric temperature and its use in multi-dimensional models, Atmos. Chem. Phys., 9, 8889-8901, doi:10.5194/acp-9-8889-2009, 2009.

Mishchenko, M. I. and Travis, L. D.: Capabilities and limitations of a current FORTRAN implementation of the T-matrix method for randomly oriented, rotationally symmetric scatterers, J. Quant. Spectrosc. Ra., 60, 309-324, doi:10.1016/S00224073(98)00008-9, 1998.

Nordh, H. L., Schéele, F. v., Frisk, U., Ahola, K., Booth, R. S., Encrenaz, P. J., Hjalmarson, Å., Kendall, D., Kyrölä, E., Kwok, S., Lecacheux, A., Leppelmeier, G., Llewellyn, E. J., Mattila, K., Mégie, G., Murtagh, D., Rougeron, M., and Witt, G.: The Odin orbital observatory, Astron. Astrophys., 402, L21-L25, 2003.

Rapp, M. and Thomas, G. E.: Modeling the microphysics of mesospheric ice particles: Assessment of current capabilities and basic sensitivities, J. Atmos. Sol.-Terr. Phy., 68, 715-744, doi:10.1016/j.jastp.2005.10.015, 2006.

Roddy, A. F.: The role of meteoric particles in noctilucent clouds, Irish Astron. J., 16, 194-202, 1984.

Sheese, P. E., Llewellyn, E. J., Gattinger, R. L., Bourassa, A. E., Degenstein, D. A., Lloyd, N. D., and McDade, I. C.: Mesopause temperatures during the polar mesospheric cloud season, Geophys. Res. Lett., 38, L11803, doi:10.1029/2011GL047437, 2011.
Stevens, M. H.: The polar mesospheric cloud mass in the Arctic summer, J. Geophys. Res., 110, A02306, doi:10.1029/2004JA010566, 2005.

Toon, O. B., Turco, R. P., Hamill, P., Kiang, C. S., and Whitten, R. C.: A One-Dimensional Model Describing Aerosol Formation and Evolution in the Stratosphere: II. Sensitivity Studies and Comparison with Observations, J. Atmos. Sci., 36, 718-736, doi:10.1175/1520-0469(1979)036<0718:AODMDA>2.0.CO;2, 1979.

Turco, R. P., Hamill, P., Toon, O. B., Whitten, R. C., and Kiang, C. S.: A One-Dimensional Model Describing Aerosol Formation and Evolution in the Stratosphere: I. Physical Processes and Mathematical Analogs, J. Atmos. Sci., 36, 699-717, doi:10.1175/1520-0469(1979)036<0699:AODMDA>2.0.CO;2, 1979.

Turco, R. P., Toon, O. B., Whitten, R. C., Keesee, R. G., and Hollenbach, D.: noctilucent clouds: Simulation studies of their genesis, properties and global influences, Planet. Space Sci., 30, 11471181, 1982.

Vergados, P. and Shepherd, M. G.: Retrieving mesospheric water vapour from observations of volume scattering radiances, Ann. Geophys., 27, 487-501, doi:10.5194/angeo-27-487-2009, 2009.

von Savigny, C., Petelina, S. V., Karlsson, B., Llewellyn, E. J., Degenstein, D. A., Lloyd, N. D., and Burrows, J. P.: Vertical variation of NLC particle sizes retrieved from Odin/OSIRIS limb scattering observations, Geophys. Res. Lett., 32, L07806, doi:10.1029/2004GL021982, 2005.

Witt, G.: Polarization of light from noctilucent clouds, J. Geophys. Res., 65, 925-933, doi:10.1029/JZ065i003p00925, 1960. 